\title{
On Generating Multicast Routes for SpiNNaker
}

\author{
Javier Navaridas, Mikel Luján, Luis A. Plana, Steve Temple and Steve B. Furber \\ School of Computer Science \\ The University of Manchester \\ Manchester, UK
}

\section{\{javier.navaridas, mikel.lujan, luis.plana, steve.temple, steve.furber\}@manchester.ac.uk}

\begin{abstract}
The human brain is an immense biological neural network characterized by high degrees of connectivity among neurons. Any system designed to simulate biologically-plausible spiking neuronal networks needs to support such connectivity and the associated communication traffic in the form of spike events. This paper demonstrates the adequacy of multicast communications to achieve such a demanding goal and introduces a collection of algorithms to generate multicast routes. These algorithms target the SpiNNaker interconnect; a two dimensional triangular toroidal mesh with support for selective multicast. As generating multicast routes is a NP-complete problem, these algorithms are an essential ingredient for an efficient operation of SpiNNaker. Although multicast networks have been studied in the literature, existing algorithms cannot be applied efficiently to SpiNNaker. A comprehensive evaluation analyzing the largest configuration of the SpiNNaker system (over 1 million ARM cores) shows that each algorithm provides diverse. benefits and drawbacks which can be exploited to avoid possible bottlenecks. Results show that the communication infrastructure of SpiNNaker will be able to support the high communication pressure exerted by simulating in realtime biologically plausible spiking neural applications
\end{abstract}

\section{Categories and Subject Descriptors}

C.1.2 [Multiple Data Stream Architectures (Multiprocessors)]: Interconnection architectures (e.g., common bus, multiport memory, crossbar switch), Parallel processors

C.1.3 [Other Architecture Styles]: Neural nets

C.4 [Performance of Systems]: Performance attributes

\section{General Terms}

Performance, Design, Theory

\section{Keywords}

Multicast route generation, Massively-parallel processing, triangular toroidal mesh, neuromimetic architecture, Spiking neural network simulation, Low-power architecture.

\section{INTRODUCTION}

The SpiNNaker neuromimetic architecture system is a biologically-inspired massively-parallel architecture based on a custommade multicore System-on-Chip (SoC). SpiNNaker targets the simulation, in biological real-time, of very large-scale spiking neural networks, with more than $10^{9}$ neurons. To put this number

Permission to make digital or hard copies of part or all of this work for personal or classroom use is granted without fee provided that copies are not made or distributed for profit or commercial advantage and that copies bear this notice and the full citation on the first page. Copyrights for third-party components of this work must be honored. For all other uses, contact the Owner/Author.

Copyright is held by the owner/author(s).

CF'14, May 20-22 2014, Cagliari, Italy

ACM 978-1-4503-2870-8/14/05.

http://dx.doi.org/10.1145/2597917.2597938 in context, it roughly represents $1 \%$ of the neurons in the human brain, or the number of neurons in the brain of a small primate [23]. Spiking neural networks communicate by means of spike events which occur when a neuron is stimulated beyond a given threshold and discharges an electrical impulse. These spikes are communicated to all connected neurons, with typical fan-outs of the order of $10^{3}$ [16]. At a realistic biological firing rate of $10 \mathrm{~Hz}$, there could be more than $10^{10}$ neuron firings per second, which can replicate up to $10^{13}$ communication events per second in the largest SpiNNaker configuration. Thus, an essential problem inherent to the simulation of spiking neural networks is how to distribute large numbers of small packets very widely amongst up to the million processors featured by SpiNNaker in an efficient way and with minimal latency.

This paper demonstrates the benefits of using a multicast architecture, rather than a more conventional unicast or point-to-point one, for this kind of application. Furthermore, given that constructing multicast routes from a source to a set of destinations is known to be an NP-complete problem [30], appropriate route generation is a crucial component of any multicast architecture. For this reason we provide a collection of algorithms to generate multicast routes for SpiNNaker which exploit the regularity of the topology. Two of the presented algorithms are based upon wellknown, oblivious routing algorithms used in point-to-point communications and have the advantage of producing multicast routes very quickly. The other two novel algorithms present an intelligent behavior as they use exploration to substantially reduce the number of network resources employed to perform the multicast communication. In the evaluations that follow, we focus on the scalability of the different algorithms and, when possible, we compare them with a unicast approach to show the benefits of multicast when handling the high-connectivity traffic generated by spiking neural networks. Finally, we show that the interconnection network of the largest SpiNNaker system can sustain neurallike traffic even in configurations that are more demanding than those anticipated.

\subsection{SpiNNaker Machines}

The construction of large-scale versions of SpiNNaker is ongoing and is expected to culminate with a 1-million core system by the end of 2014. We have already developed and fabricated some prototypes. Back in 2010 a first batch of test chips (two cores and a fully functional router) were produced and successfully demonstrated running spiking neural nets. This was followed in 2011 by the production of small quantities of full-fledged SpiNNaker chips with 18 ARM cores and the development of small boards, able to house 4 SpiNNaker chips and to support inter-board communications (see Fig. 1). These boards, due to their low-power design, have been used as control devices for robotic systems [14], providing them with real-time stimulus-response behavior [18]. The next step towards the full-fledged SpiNNaker was the production of 48-chip boards (also shown in Fig. 1) which are interconnected to form increasingly large implementations of SpiNNaker - one board forms a $10^{3}$-core machine, one 24-board card frame (right, in Fig. 1) for the $10^{4}$ one, one 5 -frame cabinet (120 boards) will 

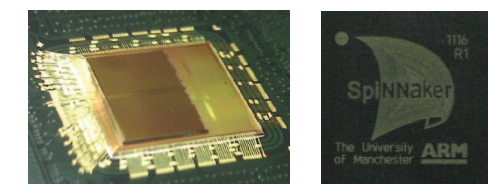

Unpackaged and packaged SpiNNaker chips.

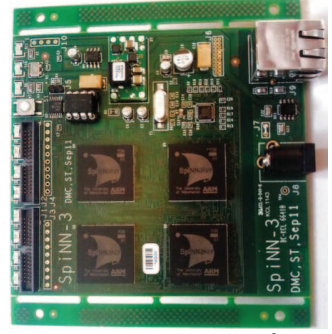

4-chip development board ( $10^{2}$ machine)

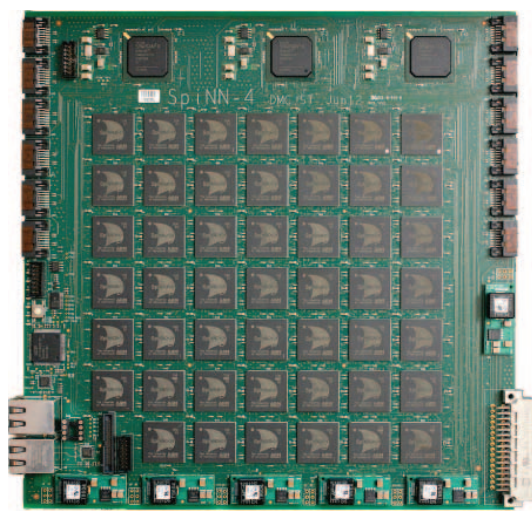

48-chip production board ( $10^{3}$ machine)

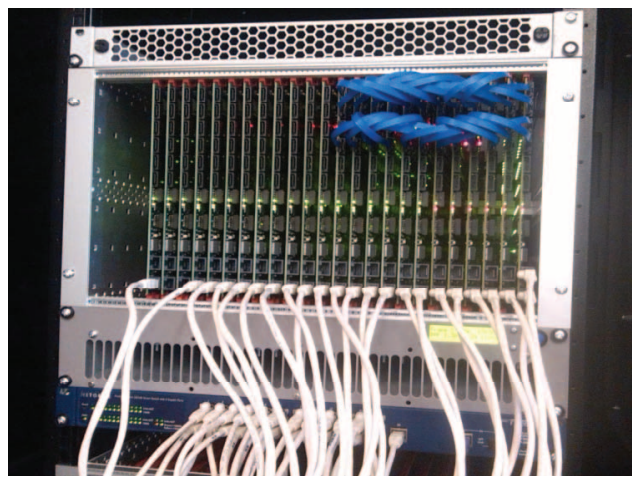

24-board frame ( $10^{4}$ machine)

Figure 1. SpiNNaker chip, experimental board with 4 chips, production board with 48 chips and 24-board card frame.

be the $10^{5}$ machine and, finally, 10 cabinets for the full-fledged, million core machine. As we approach the largest configurations of the system, the route generation is becoming an increasingly important aspect of the machine's operation

Aside from the hardware, a large collection of system software and application libraries is already offered to operate SpiNNaker, and new features are developed and released frequently. Among all the software involved in SpiNNaker it is specially important the PyNN [15] frontend which is used to define spiking neural networks and drive simulations. The PyNN/SpiNNaker combination exploits the flexibility of the SpiNNaker architecture and decouples neural applications from the actual hardware, allowing users to rapidly develop and simulate spiking neural networks without any knowledge about the intricacies of the underlying system.

\section{RELATED WORK}

\subsection{Large-scale neural systems and multicast}

The development of artificial neural network-based computing systems has remained a topic of interest for decades as it is believed they could help to understand how the brain operates - still a mystery for science. Here we review the most notable projects in this area and highlight the absence of related systems considering the use of multicast communications even when it seems to be a natural candidate due to the high connectivity shown by biological neural networks.

In the eighties and nineties the Connectionist Machine family of supercomputers came out from the MIT. Although they were designed as general purpose parallel machines, simulating neural models was one of the target applications [24]. Connectionist Machines featured specialized interconnection networks for data and control communications but did not include multicast; only a broadcast mechanism for system-level synchronization.

Later on, in the early nineties a team at U.C. Berkeley worked in the Connectionist Network Supercomputer, a system specifically tailored for neural computation [5]. The system had a target size of 128 nodes (scalable to 512) arranged as a 2D mesh. Nodes included a router to communicate but multicast was not supported, based on the expectation that the system would rely on spatial locality to improve performance. To our knowledge, a prototype of the node was built (under the codename T0), but the system never operated as a network. Experiments using up to five nodes in a bus configuration were discussed in [37].

The Blue Brain project [32] and the early stages of SyNAPSE [4] have somewhat similar applications to SpiNNaker and target simi- lar application scales. However they do not contemplate the development of a custom-made architecture, but rather rely on a general-purpose massively-parallel system: IBM Blue Gene systems [21]. This family of supercomputers is composed of tens of thousands of compute nodes, each having several PowerPC processors. Blue Gene's main interconnect is point-to-point which, as we show in this paper, might not be the most efficient way of dealing with the high connectivity of neural models.

The SyNAPSE project [17] follows a broad approach to neural modeling and is not restricted to using Blue Gene supercomputers - their stated aim is to 'develop electronic neuromorphic machine technology that scales to biological levels'. The project also involves creating custom hardware and even research into less conventional technologies such as memristors [33]. No indication has been made, however, towards investigating ways to handle the high connectivity inherent to spiking neural networks, not to mention in using multicast-based communication architectures.

Finally, the FACETS project [19] is creating a faster-than-realtime custom hardware system for simulations of large (but unspecified) size networks of biologically-inspired neurons. Its distinctive characteristic is that it relies on analogue circuits to implement neural dynamics. Communications are based on digital logic, circuit-switched synchronous communications with no support for multicast communications. Again, the high connectivity of the neural models is not addressed at hardware-level which could end up limiting the scalability of the system.

\subsection{Multicast in other areas}

Outside of the field of neural networks simulation, there is plenty of research on multicast communication strategies. However, most of those strategies were not able to be employed in SpiNNaker because they are devised with rather different purposes. To our knowledge, the most prolific areas in which research related to multicast route generation is carried out are the fields of communication networks (Internet Protocol networks, or simply IP networks) and multistage networks for high performance computing systems.

In the field of IP networks, network topologies are mostly irregular and hence the multicast algorithms are not devised to exploit network regularity, as those proposed in this paper do. In fact, in that kind of networks, topology discovery and exploration are the most significant parts of the research [8, 41].

In multistage networks for high performance computing systems the construction of multicast routes focuses on minimizing the interactions between concurrent multicast communications in order to increase network throughput $[6,11]$. Reducing network 


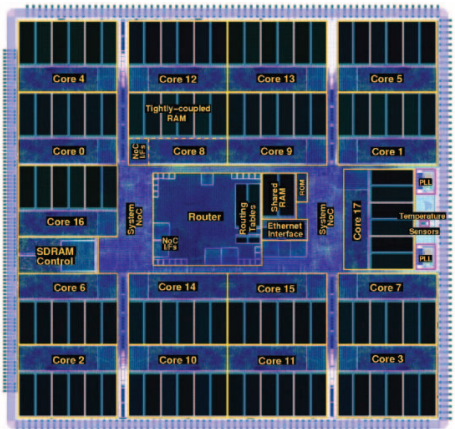

Chip Overlay
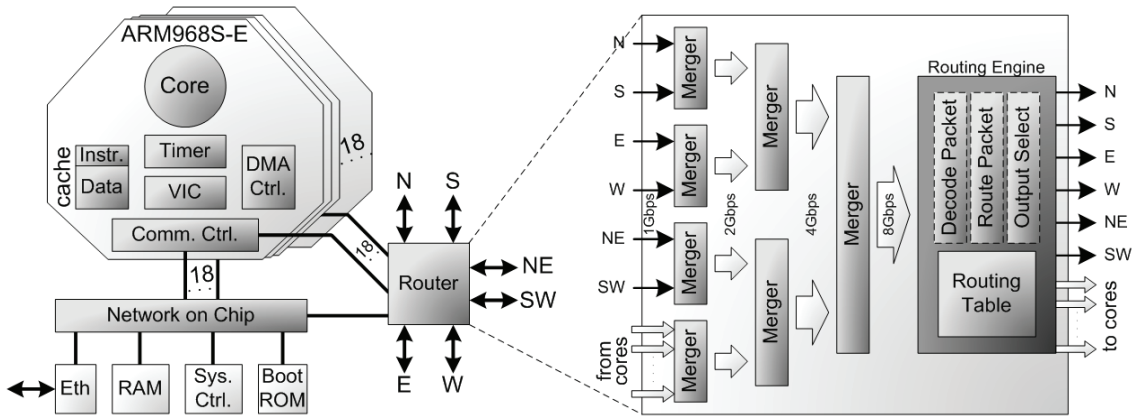

Network-centric Schematic Design

Figure 2. SpiNNaker 18-core SoC chip. utilization, however, is not considered in these areas of research. Neither is it reducing the utilization of the routing tables.

Research on multicast for mesh-like topologies as the one in SpiNNaker was quite common in the nineties [30, 36, 42] but had several limitations that discourage their use for SpiNNaker. Firstly, only wormhole switching was considered whereas SpiNNaker uses store and forward. Secondly, most proposals were based on the use of virtual channels, a facility that is not implemented in SpiNNaker due to its area constraints. Finally, the main concern of those studies was deadlock avoidance; reducing network utilization was merely a secondary issue, at best. Given that SpiNNaker already has a built-in deadlock avoidance scheme and the scarcity in terms of network resources (both routing tables and bandwidth) a different approach is desirable.

More recently, the interest on multicast communications has emerged again with the generalization of the use of Networks-onChip in MPSoCs and manycore systems [26, 43]. Some of the limitations of previous work still apply but, in addition, they require adding specific microarchitectural router features so that they are not directly applicable to SpiNNaker which, as will be discussed next, has its own architecture whose design is adjusted to very tight power and area constraints.

\section{OVERVIEW OF SPINNAKER}

The main foundations of SpiNNaker's design philosophy were to reduce power consumption, to improve reliability by means of high redundancy and to provide a flexible architecture, general enough to run a wide range of applications [38]: from the simulation of neural non-spiking neural models - e.g. Multilayer Perceptrons [39] - to a wide variety of applications completely unrelated to neural networks: discrete simulation, many-body interaction.

Each SpiNNaker SoC (depicted in Fig. 2) contains 18 low-power general-purpose ARM968 cores running an independent eventdriven neural process, which responds to events generated by different on-chip modules: timer, communications controller and DMA controller, among others. The chip is packaged together with a 128 MB SDRAM, whose primary function is to store synaptic information. Detailed simulations of the chip confirmed that each core can support up to around 1000 neurons in biological real-time [27]; we will use this figure later in our analysis.

\subsection{Communications}

Although the SpiNNaker architecture could be scaled up to 65,536 nodes, the largest SpiNNaker under construction will house 57,600 -creating a system with over a million computing cores. The nodes are interconnected using a two-dimensional triangular torus as depicted in Fig. 3. Neurons are modeled in software and their spikes generate packets that propagate through the on- and inter-chip communication fabric relying on custom-made on-chip multicast routers $[20,44]$. The primary role of this router is to direct neural event packets to those chips and cores containing destination neurons. One remarkable characteristic of the router is that it is used to perform both inter- and intra-chip communications. It has 18 ports for the cores plus six external ports to communicate with adjacent chips. To avoid the high complexity intrinsic to crossbar-based designs, SpiNNaker router uses a simpler architecture in which only one packet can use the routing engine at once (see Fig. 2 again). Hence the ports are hierarchically merged into a single pipelined queue. The routing engine is not expected to become a bottleneck as it has much higher bandwidth than the transmission ports ( $8 \mathrm{Gbps}$ vs. $250 \mathrm{Mbps}$ ). In other words, the average load of the router will be very low so router delay will barely affect the pace at which packets are processed. The router supports point-to-point and multicast communications using small packets of 40 bits. As will be shown, the multicast engine reduces the pressure at the injection ports and the number of packets traversing the network, when compared to a point-topoint alternative.

Following the Address Event Representation [31], packets do not contain any information about their destination(s), only an identifier of the neuron that has fired. The information necessary to deliver a neural packet to all the relevant cores and chips is compressed and distributed across the 1024-word routing table within each router. To allow further compression, routing tables offer a masked associative route look-up and routers are designed to perform a default routing - which requires no entry in the routing table - by sending the packet to the port opposite to the one the packet comes from, i.e. if a packet comes from the North it will be

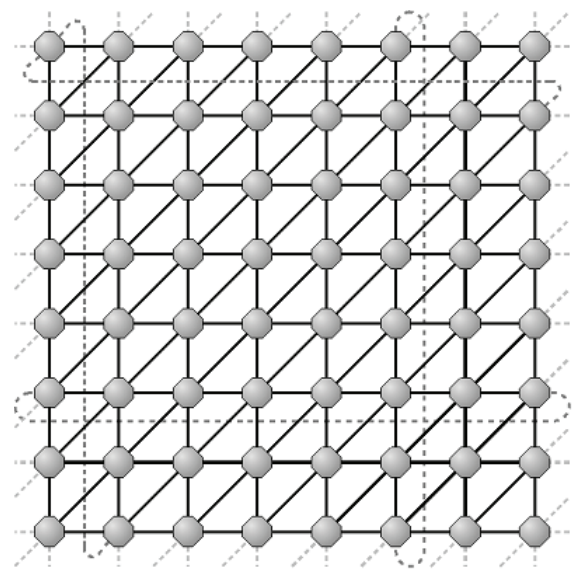

Figure 3. $8 \times 8$ instance of SpiNNaker topology. Most peripheral wrap-around links are hidden for the sake of clarity. 
Table I. Comparison between SpiNNaker and BlueGene ASICs.

$\begin{array}{lrr} & \text { Technology } & \text { Transistors } \\ \text { SpiNNaker ASIC } & 130 \mathrm{~nm} & 79 \mathrm{M} \\ \text { BlueGene/L ASIC [3] } & 130 \mathrm{~nm} & 95 \mathrm{M} \\ \text { BlueGene/P ASIC [9] } & 90 \mathrm{~nm} & 208 \mathrm{M} \\ \text { BlueGene/Q ASIC [34] } & 45 \mathrm{~nm} & 1.43 \mathrm{G}\end{array}$

sent to the South. As routing tables have a limited number of entries, keeping their utilization low is essential for the proper operation of SpiNNaker, for this reason, this will be one of the figures of merit in the following evaluations. Note that the routing tables need to be filled before starting the neural simulation and, for this reason, fast multicast routing algorithms would be preferred to reduce set-up time as long as they are able to keep network resources below the limits of SpiNNaker. Routing tables are not intended to remain static during long simulations. They can be modified dynamically in real-time to accommodate scenarios of congestion or even failures in the interconnection network. For the sake of simplicity this scenario has not been considered in the evaluations that follow.

Network flow-control is very simple. When a packet arrives at the router, one or more output ports are selected according to the information stored in the routing table and the packet is transmitted through all of them. Deadlock- and livelock-avoidance are based on packet dropping mechanisms which were studied in [35].

The design of the network fabric was scaled assuming some degree of locality of spike traffic - most destination neurons are proximate to the transmitting neuron - as is typically seen in the brain $[7,22]$. The inter-node connections were designed to support sustained bandwidths of $1 \mathrm{Gbps}$. However their self-timed nature made them more sensitive to latency than was, in principle, anticipated. This reason, together with the decision of using lowpower chip technologies has affected negatively the communications of SpiNNaker. The actual maximum link bandwidth attained by the real chip has been measured to be drastically reduced to roughly $250 \mathrm{Mbps}$. We will use this figure later on to derive the number of neurons that a system can support during our experimental work.

\subsection{Dissipated Power}

One of the main design principles which drove the development of SpiNNaker was to produce a low-power massively parallel architecture as can be seen by the nature of its components. Keeping power consumption low eases the deployment of a large system, simplifying supply and cooling requirements. It also reduces running costs which for a high-performance computer form a significant proportion of the total cost of ownership.

Each SpiNNaker chip, while the cores are quiescent, draws 360 $\mathrm{mW}$. Each processor running at $150 \mathrm{MHz}$ adds $18 \mathrm{~mW}$, therefore, with all 18 processors running, a SpiNNaker chip will consume $680 \mathrm{~mW}$ (or close to $900 \mathrm{~mW}$ at the expected $200 \mathrm{MHz}$ ). In a fullsize system this is roughly $60 \mathrm{~kW}$ of peak core power. However in neural applications it is anticipated that the processors will be asleep for a significant proportion of the time, so the actual consumption will be significantly lower. At full speed the cost of running an inter-chip link is $6.3 \mathrm{~mW}$. Therefore the consumption per bit is $2.5 \times 10-11 \mathrm{~J}$, or possibly more clearly: $40 \mathrm{~Gb} / \mathrm{J}$. With six outputs per chip and an assumed $40 \%$ utilization this adds $15 \mathrm{~mW}$ to each chip's consumption, or around $1 \mathrm{~kW}$ to the total for a full-fledged system. When the SDRAM is continuously exercised (at $130 \mathrm{MHz}$ clock) the node power is increased by $170 \mathrm{~mW}$ which represents a further $11 \mathrm{~kW}$ in a fully expanded system, proportionally less if the SDRAM duty cycle is lower than $100 \%$, as it is expected to be. The only other components of the system are a few active Ethernet ports (roughly $40 \mathrm{~mW}$ each), and ancil-

$\begin{array}{rrrr}\text { Cores } & \text { Frequency } & \text { Estimated Performance } & \text { Power } \\ 18 & 200 \mathrm{MHz} & 3,960 \mathrm{DMIPS} & 1.1 \mathrm{~W} \\ 2 & 700 \mathrm{MHz} & 2,800 \mathrm{DMIPS} & 12 \mathrm{~W} \\ 4 & 850 \mathrm{MHz} & 6,800 \mathrm{DMIPS} & 31 \mathrm{~W} \\ 18 & 1,500 \mathrm{MHz} & \text { N/A } & 55 \mathrm{~W}\end{array}$

lary devices such as LEDs, which will consume a very low amount of power relative to the rest of the system. It is safe to say therefore, that the overall peak system power should be in the range $30-75 \mathrm{~kW}$, depending on activity for a full-fledged SpiNNaker system, which means that the average consumption per chip will be in the range $0.45-1.15 \mathrm{~W}$, much lower than any current HPC technology. For instance the ASICs of Blue Gene/L and Blue Gene/P supercomputers are reported to draw $13 \mathrm{~W}$ and $30 \mathrm{~W}$ respectively $[3,9]$. This represents an order of magnitude in terms of power dissipation but their estimated performance is in par with SpiNNaker's (see Table I).

\subsection{Modeled application}

SpiNNaker is designed to simulate spiking neural networks using simple spiking neural models widely accepted by the neuroscientist community. The classic Leaky Integrate and Fire (LIF) model [28] and the more complex Izhikevich model [25] are fully supported by SpiNNaker.

Neurophysiology has provided a deep and clear understanding of the physical operation of biological spiking neurons [16]. Neurons have a membrane potential which fluctuates over time and which is affected by each received signal. Whenever an excitatory signal is received the membrane potential is increased; in contrast, if the signal is inhibitory the membrane potential is reduced. When the membrane potential exceeds a given threshold, the neuron discharges and fires a spike which affects similarly to all neurons sharing a synaptic connection, typically in the order of the thousands. The high connectivity of the neurons exacerbates the spike distribution problem.

In biological neural networks, connectivity is split into two different categories (see Fig. 4): short-range and long-range projections. Short-range projections include the connections to closely located neurons. Long-range projections connect neurons with remote areas of the brain. In general a long-range projection is connected to several neurons in the same distant area. In the experiments that follow we will use a destination distribution which is consistent with this behavior - a collection of centroids which model longrange projections located in remote areas of the network.

\section{MULTICAST ROUTE GENERATION}

In this section we describe the four algorithms to generate multicast routes considered in this research work. As explained in Section 2, we did not find any previous algorithm in the literature that can be applied directly to SpiNNaker because multicast routes are

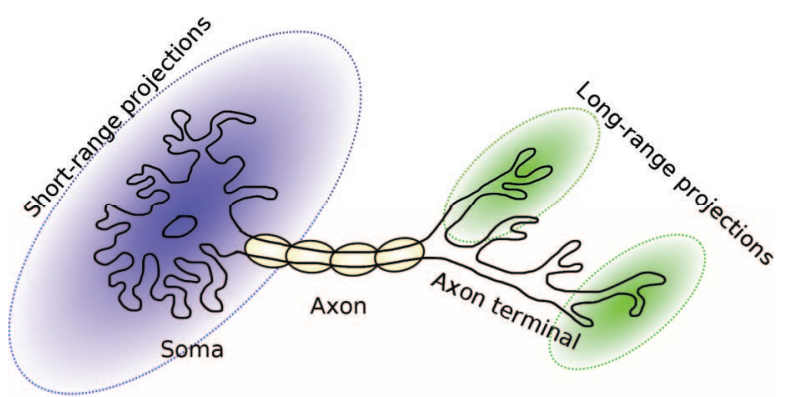

Figure 4. Example of neural communications depicting short-range (blue) and long-range (green) projections. 

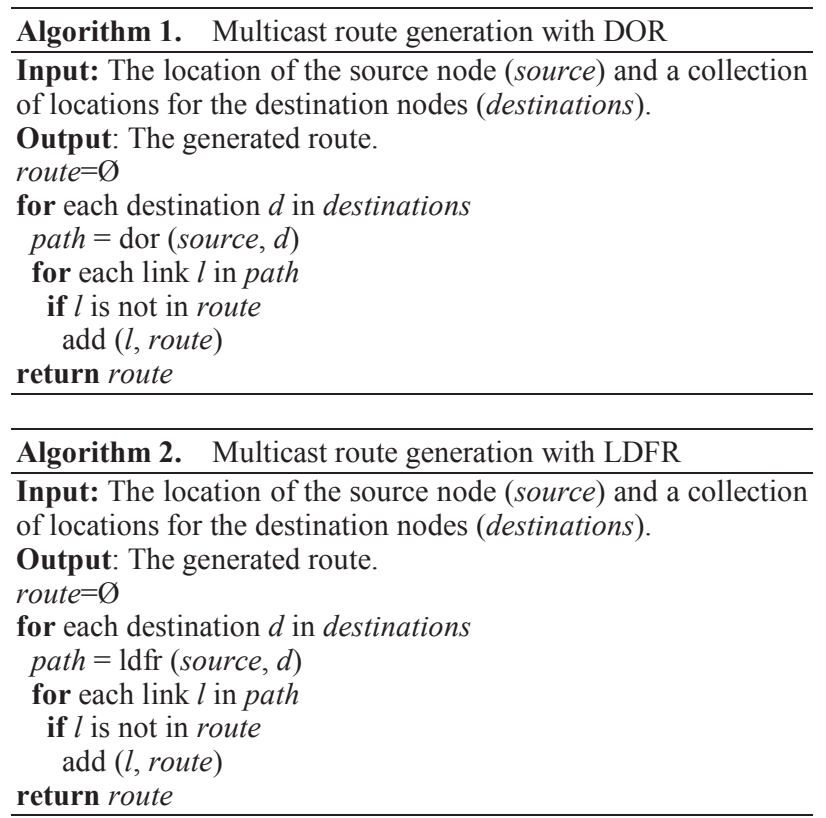

Algorithm 3. Multicast route generation with ESPR

Input: The location of the source node (source) and a collection of locations for the destination nodes (destinations).

Output: The generated route.

route $=\varnothing$

for each destination $d$ in destinations

$c=$ closest $($ source,$d)$

path $=\operatorname{ldfr}(c, d)$

for each link $l$ in path

if $l$ is not in route

add $(l$, route $)$

return route

Algorithm 4. Multicast route generation with NER

Input: The location of the source node (source) and a collection of locations for the destination nodes (destinations) sorted by their distance to source.

Output: The generated route.

route $=\varnothing$

for each destination $d$ in destinations

$\mathrm{s}=$ surroundings $(\mathrm{d})$

path $=\operatorname{ldfr}(s, d)$

for each link $l$ in path

if $l$ is not in route

add $(l$, route $)$

return route

generated often with rather different objectives and restrictions.

For this reason our starting point was to consider and to adapt oblivious routing algorithms typically used in unicast interconnects [12]. Dimension order routing (DOR) which is typically the baseline multicast routing in cube-like topologies [29] was our first approach. Further we implemented longest dimension first routing (LDFR) another well-known algorithm and a good candidate because it favors route overlapping. Also, we devised two novel, more sophisticated algorithms which make intelligent decisions by exploring how to connect different branches of the multicast tree to avoid inefficient parallel branches. Note that the algorithms presented here are focused on the triangular torus topology used by SpiNNaker (see Fig. 2 again), but are not restricted to it.
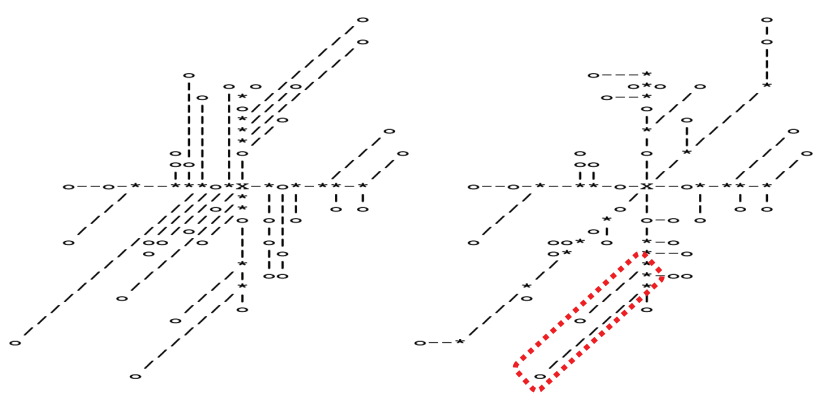

a) Dimension Order Routing

b) Longest Dimension First Routing
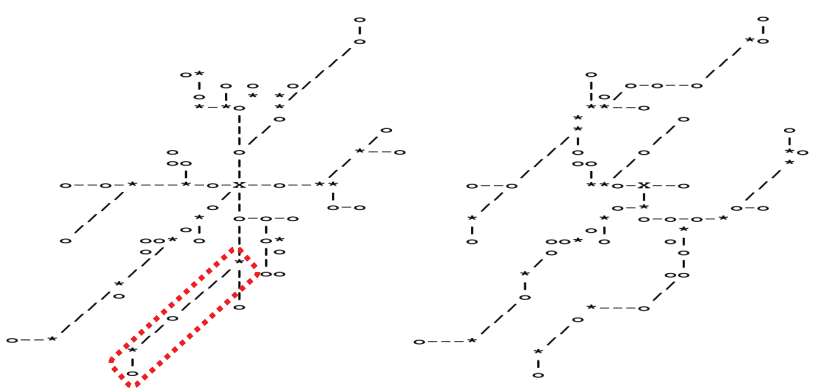

c) Enhanced Shortest Path Routing

d) Neighbour Exploring Routing

Figure 5. Example of multicast trees for each algorithm.

Legend: ' $x$ ' source, ' 0 ' destinations, ' $*$ ' an entry is required

in routing table, '-' '|' '//' default route (no entry needed)

The proposed algorithms are general enough to be applied in any mesh-like topology; e.g. the 3D torus topologies implemented in IBM's BlueGene/L [1] and BlueGene/P [21] and Cray's XT supercomputers [40], the 5D topology in IBM's BlueGene/Q [34] or the $6 \mathrm{D}$ tofu interconnect in the $\mathrm{K}$ computer [2]

In the algorithmic definitions below, all the functions have comprehensible names and will not be discussed unless required for a proper explanation of the algorithm. In all cases, 'source' represents the node for which the multicast route is being generated, 'destinations' contains the destination set and 'route' stores the multicast route under construction. The operation of all the proposed algorithms is similar; they start with an empty route and build the multicast route by adding links in a destination-bydestination basis as determined by the algorithm. Exemplar multicast routes are shown in Fig. 5.

Dimension Order Routing (DOR) is, by far, the most common routing strategy used in mesh-like interconnection networks. For this reason it was the first algorithm implemented in SpiNNaker and the one that has been used in the small-scale simulations run up to date (using a few 4-chip boards). However, as we will see later, this algorithm may not be adequate because of the high number of parallel branches (see Fig. 5). For this reason we aim to replace it with a better suited routing generation algorithm for scaled up versions of SpiNNaker. DOR description is very simple: follow a path from the source to each destination travelling dimensions in order (first advance through $\mathrm{X}$, then through $\mathrm{Y}$ and then through the Diagonal). Note that only shortest paths are considered and that most communications will share the hops in dimension $\mathrm{X}$ or $\mathrm{Y}$. The algorithmic definition of this strategy is shown in Algorithm 1. Function 'dor' returns the collection of links traversed from 'source' to ' $d$ ' using dimension order.

Longest Dimension First Routing (LDFR) is the first replacement for DOR implemented in SpiNNaker [13]. It uses another classic algorithm which also ensures shortest paths. In LDFR, routes are generated travelling first in the dimensions with more hops. Note that if two dimensions have the same number of hops, one of them 
will be chosen at random. The definition of this strategy (see Algorithm 2) is similar to DOR's, but using a different routing function, 'Idfr' which returns the links traversed using longest first routing from 'source' to ' $d$ '.

Enhanced Shortest Path Routing (ESPR) is a more complex strategy which uses shortest path routes only. Each destination ' $d$ ' looks for the closest node ' $c$ ' reachable using a shortest path towards the source and adds the route from ' $c$ ' to ' $d$ ' (using longest first routing) to the solution. If there is no intermediate node, then ' $c$ ' will be the source. ESPR creates similar multicast routes to those produced by the LDFR strategy but reduces the number of inefficient parallel branches when several nodes are close from each other as can be seen clearly in the bottom branches of Fig. 5b and $5 \mathrm{c}$ (marked in red). Algorithm 3 shows ESPR's pseudo-code.

The last strategy, called Neighbor Exploring Routing (NER), is the most complex of the presented algorithms. The destinations are sorted from the closest to the furthest. Then each destination ' $d$ ' looks for the closest route point ' $s$ ' (the source, an already considered destination or a bypassed node) in its surroundings and adds the route between ' $s$ ' and ' $d$ ' to the solution. This way, as each node is connected to its closest route point, the requirements in terms of network resources should be reduced drastically. Algorithm 4 shows the pseudo-code definition of this strategy. Function 'surroundings' performs the search for the closest route point. It explores around a destination (first at distance 1, then 2, and so on) in the already generated part of the multicast route to try to find a connecting point. We have implemented a threshold distance $M$ which will connect the point to the source if no other point is found at that distance. In the following experiments we used an $M$ of 10 .

\section{EXPERIMENTAL WORK}

From the example multicast trees shown in Fig. 5 it is already apparent the differences in terms of number of network resources employed by each of the algorithms. We will proceed now to evaluate how they will behave when generating routes for the largest SpiNNaker configuration $(256 \times 256$ nodes $)$ and workloads covering far beyond the most extreme scenarios expected. As discussed in Section 3, there are several important figures of merit: (i) compute time required to construct the multicast routes, (ii) use of network resources, (iii) balanced use of these resources, (iv) number of entries in the routing tables, and (v) number of neurons that can be supported by the interconnection network.

In the analyses that follow we show average values obtained from executing the algorithms for 200,000 different configurations for

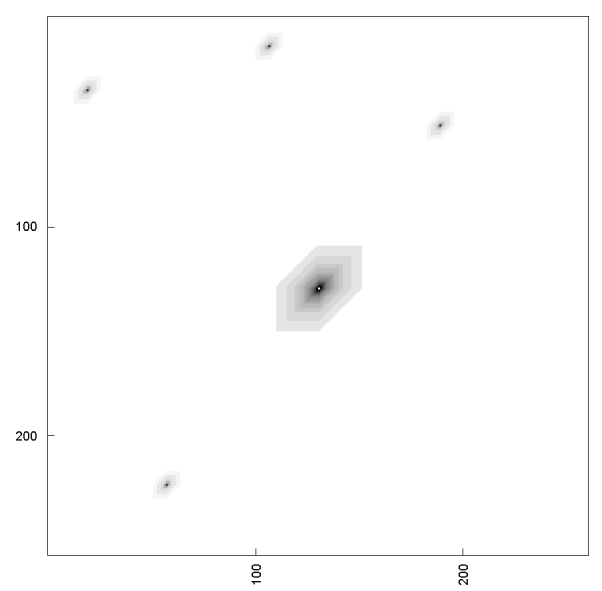

Figure 6. Example of a distribution with 4 centroids. Darker means higher probability of selecting a destina- each centroid/destination tuple. The experimental platform was an Intel ${ }^{\circledR}$ Core $^{\mathrm{TM}}$ i7 M 640 with 8 GB of RAM.

\subsection{Traffic Model}

Using traffic from actual neural applications would be our preferred way to perform this evaluation. However neural applications of such magnitude $\left(10^{9}\right.$ neurons $)$ are not readily available. For this reason, we have devised a centroid-based traffic model which resembles neural communications (as explained in Section 3.3) and, in addition, provides a flexible workload which allows varying the degree of locality of communications - the more centroids we use the less local the traffic is.

Each centroid defines the centre of a long-range projection and has a $5 \%$ of probability of being chosen by destinations. We studied models having from 0 to 10 centroids, which in turn allows from $100 \%$ to $50 \%$ probability of destinations being short-range projections. Once a destination is assigned to a centroid or to the area around the source it selects randomly a node using an exponential distribution of the distance, so that closer nodes are more likely to be selected. Selecting the same node more than once is not permitted, so if a node has been selected before, a new destination will be chosen.

The location of the centroids is distributed uniformly around the system with only one restriction: they have to be located at a distance of, at least, 32 hops from the source node. This way we ensure that the centroids are actually in a remote area of the network. We will study configurations having within 1 and 1024 different destination nodes. Given the high number of neurons we can multiplex within a chip, the high locality nature of the traffic and the neural network generation procedure implemented in SpiNNaker [13] the number of destination nodes should not exceed the tens very often. The cases with above one hundred destination nodes should be considered worst-case.

Figure 6 shows a simple example with 4 centroids. As each of these have a $5 \%$ probability of being selected, the big area around the source (in the centre of the figure) will attract an $80 \%$ of the destination nodes.

\subsection{Generation time}

One of the most important characteristics we consider in this evaluation is the time required to generate a multicast route because it has a great influence on the time an application requires to start being executed on top of SpiNNaker. We have studied how the execution time required by each of the algorithms scales with respect to the number of destinations and the number of centroids. Fig. 7 shows the average time required to compute a multicast route as we increase the number of destination nodes and the number of centroids of our model. For the sake of simplicity, we show only the specific cases of the evolution of the execution time for the 10-centroid collections of destinations and the evolution of the number of centroids for the 128-node collection of destinations. The results obtained in the rest of scenarios are consistent with those displayed here and are not shown for the sake of simplicity.

The results show how the time required to compute a multicast route is greatly affected by the number of destination nodes independently of the algorithm. In all cases the execution time increases super linearly, which may render configurations in which destination neurons are placed in lots of different chips intractable. Regarding the number of centroids, they affect the time needed to generate the multicast routes but sub linearly, so this figure does not affect the execution time to the same extent that the number of destinations. Analyzing the different algorithms we can see how in general the DOR and LDFR are executed faster than the more complex ESPR and NER, up to an order of magni- 

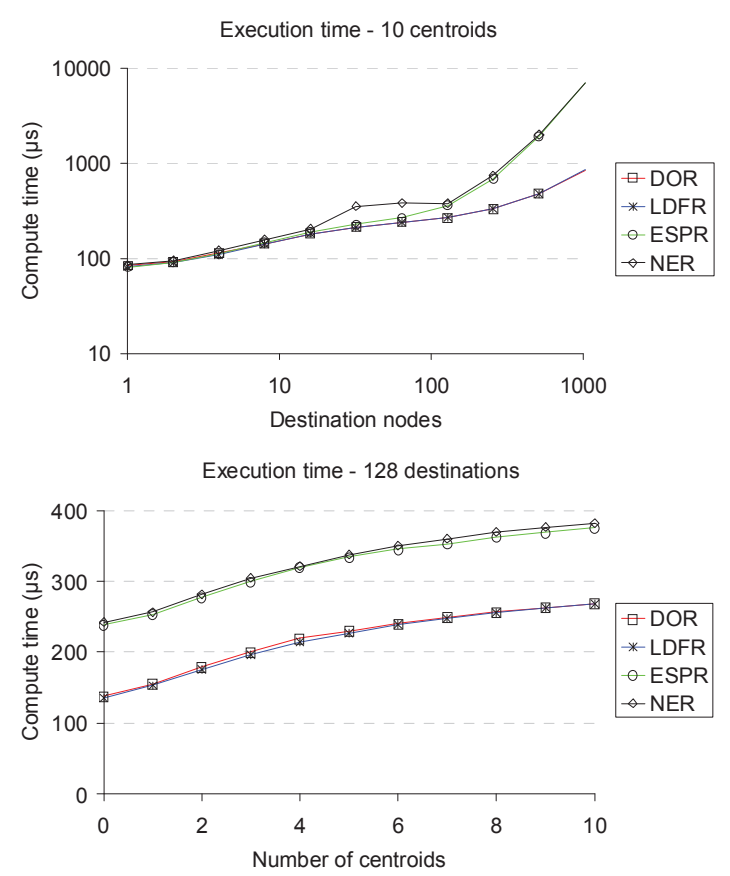

Figure 7. Impact of the number of destination nodes (top) and centroids (bottom) on the time needed to generate a multicast tree with each algorithm.

tude. Furthermore they have better scalability as the differences are more noticeable for larger number of destinations.

\subsection{Utilization of network resources}

Other important characteristic to evaluate is how the different algorithms make use of the network resources of SpiNNaker. As explained before, the low-power design of SpiNNaker limited the performance of the communications. For this reason, reducing the use of network resources is a priority to avoid the network from becoming congested, which would lead to packet dropping levels far beyond those acceptable for the execution of the target application[35]. Fig. 8 shows the link traversals employed by each algorithm to communicate a spike to all connected destinations. Again we show how this figure increases with the number of destination nodes and the number of centroids. For the sake of completeness, we show also the link traversals that would be employed by a unicast alternative. In the figure, the benefit of using a multicast communication scheme is readily evident because the amount of resources required by unicast communications can be more than one order of magnitude larger than those employed by the worst multicast alternative. In a unicast scheme, the network resources increase linearly with the number of nodes whereas with the multicast schemes they increase less than linearly. Regarding the number of centroids, it is clear that it affects sub linearly both the multicast and the unicast schemes. If we compare the different algorithms, we can see that DOR is the one requiring more network resources, then LDFR and ESPR and finally, with a significant difference, NER.

However, apart from the total amount of resources employed it is also important that the multicast trees use the network in a balanced way because an unbalanced use of one of the dimensions may lead to this dimension becoming a bottleneck; in other words, an algorithm with a lower overall use of resources but a less balanced use of them may perform worse than a algorithm using more resources in a more balanced way. For this reason Fig. 9 shows the network resources per dimension employed by each
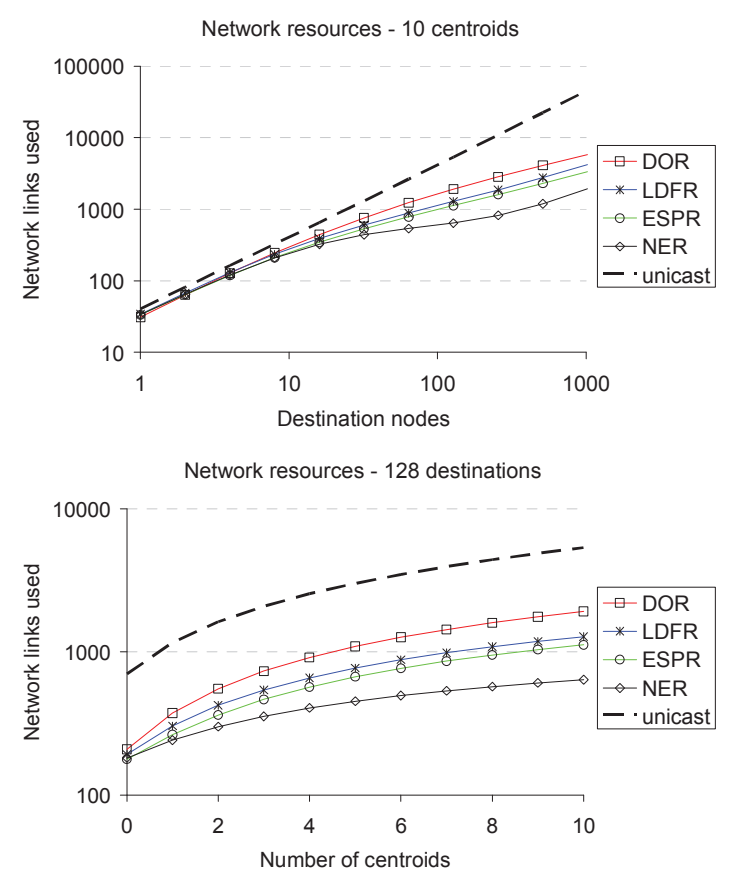

Figure 8. Impact of the number of destination nodes (top) and the number of centroids (bottom) on the number of network resources employed by each algorithm.

algorithm. In the plot we can see how all algorithms except DOR provide a reasonably balanced use of the dimensions. The extreme differences in the utilization of the dimensions by DOR-generated routes will translate into the diagonal becoming a bottleneck which in turn will lead to the interconnection network operating suboptimally. Finally, it is worth noticing that LDFR is the one providing the most balanced utilization of the dimensions.

\subsection{Utilization of the routing tables}

As discussed before, the routing tables have a very limited number of entries and therefore they are an important resource of SpiNNaker. Keeping the number of routing entries low is essential for the operation of SpiNNaker. Fig. 10 show how the number of routing entries employed by each algorithm scales with the number of destinations and the number of centroids. As in the preceding subsections, the results of non-plotted experiments are consistent with the ones displayed in the plots. The results show how all the algorithms seem to scale roughly linearly with the two parameters, being DOR the one with worse scalability as, in both cases, it is the one requiring the least entries when the number of destinations or centroids are low, but is the one requiring the most entries with the largest values of these parameters. In general we

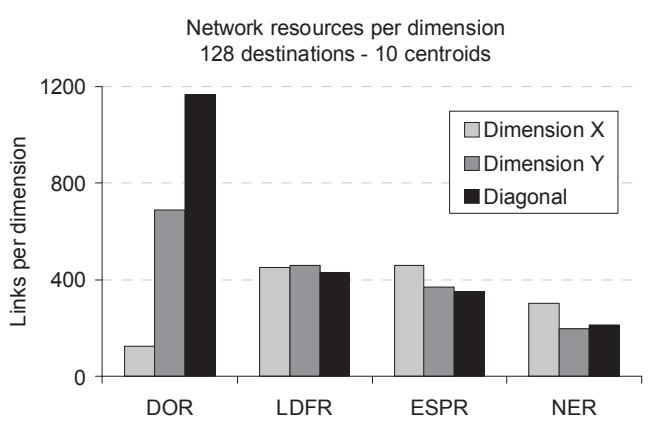

Figure 9. Per dimension use of network resources. 

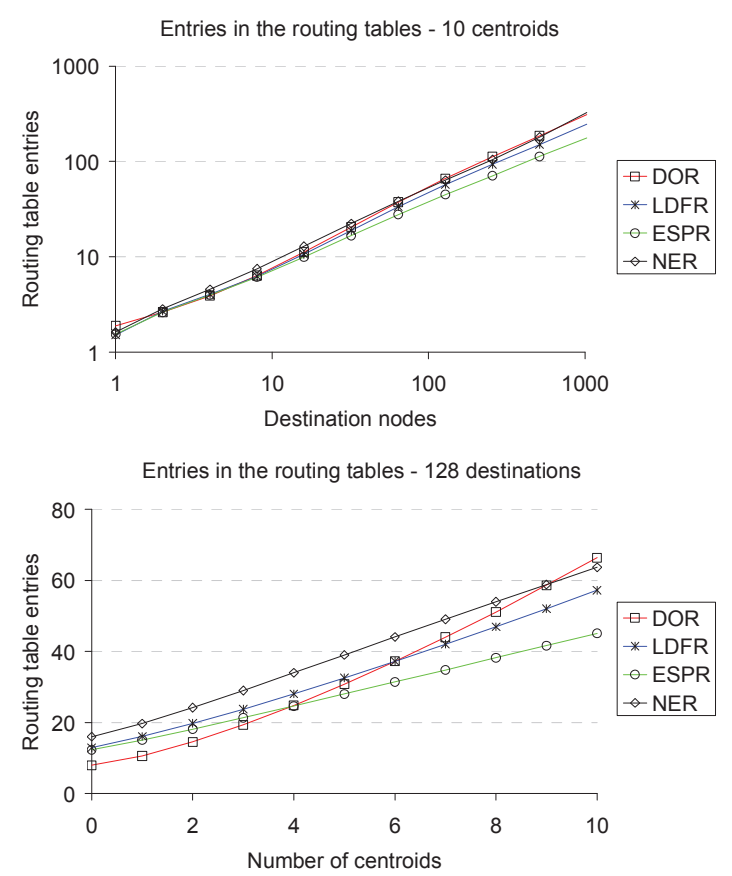

Figure 10. Impact of the number of destination nodes (top) and the number of centroids (bottom) on the number of routing entries employed by each algorithm.

can see how ESPR is the algorithm using the lowest number of routing entries in most of the cases. For this reason, it will be the preferred algorithm in those cases in which reducing the utilization of this resource becomes essential to accommodate the workload.

\subsection{Simulable neurons}

To conclude our experimental work we will compare the number of neurons the system can support with each algorithm. This is an important figure of merit because if the network becomes the limiting factor for simulation, SpiNNaker will not be able to operate efficiently or to scale up to the expected neural network sizes. We derive the number of neurons per node supported by the network $\left(n_{n}\right)$ as the per-node bandwidth (the number of links, $L$, multiplied by the link bandwidth, $B_{L}$ ) divided by the bandwidth used by each neuron (the number of link traversals per spike, $N$, multiplied by the firing rate, $f_{s}$, and by the packet size in bits, $p_{s}$ ):

$$
n_{n}=\frac{B_{L} \cdot L}{N \cdot f_{s} \cdot p_{s}}
$$

So that, considering the maximum firing rate expected in biological systems $(10 \mathrm{~Hz})$, the number of links (6) and the link traversals as shown before we can infer an upper limit for the number of neurons supported by the network. To see when the network becomes a limiting factor we consider the limit imposed by the cores (typically 16 application cores simulating $1 \mathrm{~K}$ neurons each) as an upper bound for the amount of neurons that can be simulated. Fig. 11 shows the minimum between these two limits (16K and $n_{n}$ ).

For the sake of completeness we will also consider the use of unicast to distribute the spike events. The considered unicast systems include the SpiNNaker router using point-to-point packets only and a few high-performance point-to-point architectures: the router in the Blue Gene/L (1.4 Gbps, 64-bit packets) [1], the router in the Blue Gene/P (3.2 Gbps, 64-bit packets) [3], the SeaStar router (2.2 Gbps, 64-bit packets) [10] and the SeaStar2+ router (6 Gbps, 64-bit packets) [40]. Note that all of these are general pur-
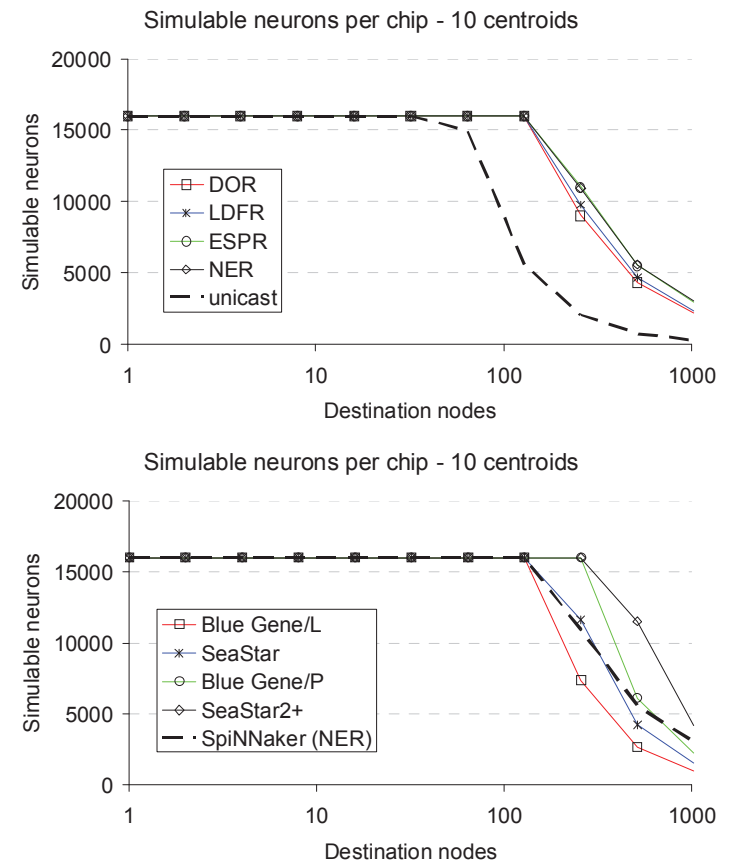

Figure 11. Impact of the number of destination nodes on the neurons simulable per SpiNNaker chip (top) and comparison with high performance unicast architectures (bottom).

pose routers whose design did not consider the high connectivity of neural applications and that they would not be able to be implemented in SpiNNaker due to area and power restrictions.

In the plots we can see how the use of multicast noticeably increases the number of simulable neurons in SpiNNaker as a unicast approach with the specifications of the on-chip router would reduce the number of neurons that can be simulated as we increase the fan-out. Furthermore, we can see how the multicast algorithm shows reasonable performance levels when compared with high performance point-to-point routers. Only the SeaStar2+ router outperforms clearly the multicast SpiNNaker router and this is at a cost of having 24 times faster connections (250 Mbps versus $6 \mathrm{Gbps})$. Notice how the network will only become a bottleneck in extreme scenarios in which the number of destination nodes exceeds the hundreds which, as discussed, is quite unlikely.

\section{DISCUSSION OF RESULTS}

From the previous results we can observe that DOR allows constructing multicast routes very quickly but, in turn, employs a large number of network resources and may require an excessive number of routing table entries. A particularly negative aspect of DOR is that the utilization of the different dimensions of the topology is very unbalanced so that the diagonal (the one suffering the most traffic pressure) may become a communication bottleneck. These negative characteristics motivate the rejection of DOR as other algorithms outperform it in all aspects.

LDFR can generate multicast trees as fast as DOR, but drastically reduces the requirement in terms of network resources and number of entries in the routing tables. However the main advantage of LDFR is that it produces the most balanced utilization of the dimensions so none of them will become a bottleneck. In general, we can state that this is a well-rounded alternative for generating multicast routes, which motivated its implementation [13].

ESPR reduces the network resource requirements when compared with DOR and LDFR and keeps balanced the per-dimension utili- 
zation. Furthermore it is the algorithm requiring the least entries in the routing tables so it is possible to use this algorithm when reducing the number of routing entries is essential for an adequate operation of SpiNNaker. The cost to pay is increasing the time required to produce the multicast routes.

Finally, NER produces the multicast routes using the lowest number of network resources. Its main disadvantages are that the multicast trees are produced very slowly and that it may require a larger number of entries in the routing tables than LDFR and ESPR. For this reason NER can be employed to generate multicast routes in those cases in which reducing network utilization is critical and the number of entries in the tables is not.

In general, we can state that we have provided a flexible collection of multicast route generation algorithms which can be employed in different situations depending on which kind of network element bounding the simulation. The recommended doctrine would be to use LDFR as to generate routes quickly and swap to ESPR or NER if either the number of entries in the routing tables or the network capacity becomes the limiting factor.

To conclude, we have shown how the use of multicast reduces considerably the utilization of the network when compared with unicast. For this reason, the modest-specification multicast router in SpiNNaker is able to support the communication phases of neural communications and thrown performance levels comparable to much more powerful unicast routers with connections more than one order of magnitude faster than SpiNNaker's. This is because the high fan-out nature of synaptic connectivity can be efficiently handled by a multicast communications architecture as the one implemented in the system.

\section{CONCLUSIONS AND FUTURE WORK}

In this paper we have highlighted the importance of multicast routing for the spiking neural networks executed on SpiNNaker in which each neuron must communicate its activation to thousands of other neurons. In the largest SpiNNaker configurations this generates a demand of more than $10^{13}$ communication events per second.

Our analysis shows that the low-spec, custom-made multicast interconnection network will be able to sustain this demand in most foreseeable situations. Only in unlikely scenarios, with extremely low locality and pathological allocation of neurons, will the network become a bottleneck. A multicast approach requires noticeably less network bandwidth than a unicast approach and therefore it is able to support the simulation of a significantly larger amount of neurons. More specifically, we have shown how the multicast router implemented in SpiNNaker can achieve better performance than more complex and high-spec point-to-point router designs such as IBM BlueGene/L's router or Cray SeaStar router, even though they provide much higher link bandwidth.

The main contribution of this paper is the description of four routing algorithms to construct multicast trees for mesh-like topologies as the one in SpiNNaker and evaluated them in a comprehensive number of performance metrics related to the most sensitive resources of SpiNNaker's communications infrastructure. Our results show that generating multicast routes using DOR, one of the most frequently used algorithms for mesh-like topologies, and the first one implemented on SpiNNaker, is counterproductive because it demands the highest network resources both in terms of bandwidth and routing entries. This discovery led to substitute this algorithm, in SpiNNaker, by LDFR, which proved to be a well-rounded solution as the multicast routes can be generated very quickly while keeping resource requirements low and balanced.

More sophisticated strategies, which look for routes in their surroundings, have been also considered. ESPR searches for connec- tions using always shortest paths; in contrast NER searches in all the surroundings even if no shortest path is used. These two strategies further reduce the network requirements but, as they generate multicast routes noticeably slower than the oblivious strategies, they will be used only in those cases in which any of the scarce resources of the network (either bandwidth or routing tables) limits system scalability. Furthermore, as they feature more intelligent behavior, it is possible to make them aware of network failures to adapt routes in order to avoid using faulty areas of the network. This will be an essential characteristic of multicast route generation algorithms once large-scale versions of the system are constructed as they are likely to suffer from failures and being able to adjust to these failures and tolerate them is key for proper operation.

In this paper these four mechanisms have been described and implemented in the context of the SpiNNaker topology, but they are general enough to be employed in other mesh-like topologies. For example we could adapt the presented algorithms to the 3D tori implemented in state-of-the-art massively parallel processors such as the IBM's BlueGene or the Cray's XT families. This adaptation is straightforward simply by using $3 \mathrm{D}$ torus routing functions while keeping the strategy formulations, as they can use the diagonal as the $\mathrm{Z}$ dimension of a $3 \mathrm{D}$ topology. Furthermore, the obtained results do not depend heavily on the topology and for this reason they can be extrapolated to other mesh-like network configurations safely.

As a future line of research we plan to explore new methodologies to generate multicast routes for SpiNNaker. These methodologies will include the utilization of optimization and clustering techniques in order to generate the multicast routes for SpiNNaker and also to allocate the neurons into SpiNNaker in such a way that distances among communicating neurons are as short as possible and the number of destination nodes is reduced to improve the efficiency of the multicast route generation. However the application of such techniques will be conditioned to the availability of fast algorithms capable of generating optimized multicast routes within reasonable times. They will be of special importance once large-scale SpiNNaker systems are constructed.

\section{ACKNOWLEDGMENTS}

The Spinnaker project is supported by the Engineering and Physical Sciences Research Council, through Grant EP/G015740/1, and also by ARM. Dr Luján holds a Royal Society University Research Fellowship.

\section{REFERENCES}

[1] Adiga, N. R., et al. "An Overview of the BlueGene/L Supercomputer". In Proc. of the ACM/IEEE Supercomputing conference. Baltimore, MD, USA, 60:1-60:22. 2002.

[2] Ajima, Y., et al "The Tofu Interconnect". In Proc. of the IEEE 19th Annual Symposium on High Performance Interconnects, Santa Clara, CA, USA 87-94. 2011.

[3] Alam, S., et al. "Early evaluation of IBM BlueGene/P". In Proc. of the ACM/IEEE Conference on Supercomputing, Austin, TX, USA, 1-12. 2008.

[4] Ananthanarayanan, R., et al " The cat is out of the bag: cortical simulations with $10^{9}$ neurons, $10^{13}$ synapses”. In Procs. of Conf. on High Performance Computing Networking, Storage and Analysis, SC '09, New York, NY, USA, 63:1-63:12.

[5] Asanovic, K., et al " A supercomputer for neural computation”. In Proc. 1994 Intl. Conf. on Neural Networks (ICNN94). Orlando, FL, 5-9. 
[6] Bhattacharya, S., et al. "Multicasting in Generalized Multistage Interconnection Networks". Journal of Parallel and Distributed Computing, 22, 1, 80-95. 1994.

[7] Binzegger, T., Douglas, R. J., and Martin, K. A. "Topology and dynamics of the canonical circuit of cat V1". Neural Networks, 22, 8, 1071-1078. 2009.

[8] Breitbart, Y., et al. "Topology discovery in heterogeneous IP networks: the NetInventory system". Transactions on Networking, 12, 3, 401-414. 2004.

[9] Bright, A. A., et al. "Creating the BlueGene/L supercomputer from low-power SoC ASICs". IEEE Intl Solid-State Circuits Conference. 2005. San Francisco, CA, 188-189.

[10] Brightwell, R., Pedretti, K., and Underwood, K. D. "Initial performance evaluation of the Cray SeaStar interconnect". In Proc. of the 13th Symposium on High Performance Interconnects, Stanford, CA, 51-57. 2005.

[11] Coll, S., et al. "Efficient and Scalable Hardware-Based Multicast in Fat-Tree Networks". IEEE Transactions on Parallel and Distributed Systems, 20, 9, 1285-1298. 2009.

[12] Dally, W. J., and Towles, B. "Principles and Practices of Interconnection Networks". Morgan Kaufmann Series in Computer Architecture and Design. 2004.

[13] Davies, S., et al. "Population-Based Routing in the SpiNNaker Neuromorphic Architecture". In Proc. of the 2012 Intl Joint Conf on Neural Network, Brisbane, Australia.

[14] Davies, S., et al. "Interfacing real-time spiking I/O with the SpiNNaker neuromimetic architecture". Australian Journal of Intelligent Information Processing Systems 11(1), 2011.

[15] Davison, A., et al. "PyNN: a common interface for neuronal network simulators". Front. Neuroinform. 2, 11. 2008.

[16] Dayan, P., and Abbott, L. "Theoretical Neuroscience". Cambridge: MIT Press, 2001.

[17] Defense Sciences Office. "Systems of neuromorphic adaptive plastic scalable electronics (SYNAPSE)". 2011.

[18] Elliott, T. and Shadbolt, N. "Developmental robotics: Manifesto and application". Philosophical Trans. Royal Soc., A, 361. 2003.

[19] Fieres, J., Schemmel, J., and Meier, K. “ Realizing biological spiking network models in a configurable wafer-scale hardware system". In Procs. of the 2008 IEEE Intl Joint Conf on Neural Networks, Hong Kong, 969-976.

[20] Furber, S. B., Temple, S., and Brown, A. " On-chip and inter-chip networks for modelling large-scale neural systems". In Procs. of the Intl. Symposium on Circuits and Systems, ISCAS-2006, Kos, Greece, 1945-1948

[21] Gara, A., and Moreira, J. "IBM Blue Gene supercomputer". IBM Research Report. 2011.

[22] Hagmann, P., et al. "Mapping the Structural Core of Human Cerebral Cortex". PLoS Biology, 6, 7, e159. 2008.

[23] Herculano-Houzel, S. "The human brain in numbers: a linearly scaled-up primate brain". In Front. Hum. Neurosci., 3:31. 2009.

[24] Hillis W.D. and Tucker, L.W. "The CM-5 Connection Machine: a scalable supercomputer". Commun. ACM 36, 11 (Nov 1993), 31-40.

[25] Izhikevich, E. "Simple model of spiking neurons". Trans. on Neural Networks 14, 1569-1572. 2003.

[26] Jerger, N. E., Peh, L. S., and Lipasti, M. “ Virtual Circuit Tree Multicasting: A Case for On-Chip Hardware Multicast
Support”. In Procs of the 2008 International Symposium on Computer Architecture, Beijing, 229 - 240.

[27] Jin, X., Furber, S. B., and Woods, J. V. "Efficient Modelling of Spiking Neural Networks on a Scalable Chip Multiprocessor". In Procs. of the 2008 International Joint Conference on Neural Networks, Hong Kong, 2812 - 2819.

[28] Koch, C., and Segev, I. "Methods in Neuronal Modeling". The MIT Press. 1989.

[29] Lin, X., McKinley, P.K. and Ni, L.M. "Deadlock-free multicast wormhole routing in 2-D mesh multicomputers". Parallel and Distributed Systems, IEEE Transactions on, vol.5, no.8, pp.793-804, Aug 1994.

[30] Lin, X. and Ni, L.M. "Multicast communication in multicomputer networks". Parallel and Distributed Systems, IEEE Transactions on, 4 (10), pp.1105-1117, Oct 1993.

[31] Maas, W., and Bishop, C. M. "Pulsed neural networks". The MIT Press. 1998.

[32] Markram. H. “The Blue Brain Project”. Nature Revs. Neuroscience 7, 2, 153-160. 2006.

[33] Merolla, P., et al. "A digital neurosynaptic core using embedded crossbar memory with $45 \mathrm{pJ}$ per spike in $45 \mathrm{~nm}$ ". Custom Integrated Circuits Conference, San Jose, CA, 2011.

[34] Milano, J., and Lembke, P. "IBM System Blue Gene Solution: Blue Gene/Q Hardware Overview and Installation Planning”. IBM Red Books. 2012.

[35] Navaridas, J., et al. "Understanding the Interconnection Network of SpiNNaker". Procs of the 23rd Intl Conf on Supercomputing, York Town Heights, NY, 2009

[36] Panda, D.K., Singal, S. and Kesavan, R. "Multidestination message passing in wormhole k-ary n-cube networks with base routing conformed paths". Parallel and Distributed Systems, IEEE Transactions on , 10(1), pp.76-96, Jan 1999

[37] Pfaerber, P., and Asanovic, K. " Parallel neural network training on multispert". In Procs. IEEE 3rd Intl Conf on Algorithms and Architectures for Parallel Processing (ICA3PP'97). Melbourne, Vic., 659 - 666.

[38] Rast, A. D., et al. "Managing burstiness and scalability in event-driven models on the SpiNNaker neuromimetic system". Intl Journal of Parallel Programming 40(6), 2011.

[39] Rosenblatt, F. "Principles of Neurodynamics: Perceptrons and the Theory of Brain Mechanisms". Washington, Spartan Books. 1961.

[40] Sadaf, A. R., et al. "Cray XT4: an early evaluation for petascale scientific simulation". In Proc. of the 2007 ACM/IEEE Conference on Supercomputing, Reno, NV, USA, 1-12.

[41] Shapiro, et al. "Topology discovery service for routerassisted multicast transport". IEEE Open Architectures and Network Programming, New York, NY, 2002.

[42] Tseng; Y-C., Panda, D.K. and Lai, T-H. "A trip-based multicasting model in wormhole-routed networks with virtual channels". Parallel and Distributed Systems, IEEE Transactions on , 7(2), pp.138-150, Feb 1996.

[43] Wang, L., et al. " Recursive partitioning multicast: A bandwidth-efficient routing for Networks-on-Chip". In Procs of the International Symposium on Networks-on-Chip . Washington, DC, USA, 2009.

[44] Wu, J., and Furber, S. B. "A Multicast Routing Scheme for a Universal Spiking Neural Network Architecture". The Computer Journal, 53, 3, 280-288 